\title{
Alzheimer's disease hypothesis and related therapies
}

Xiaoguang Du ${ }^{1}$, Xinyi Wang ${ }^{1}$ and Meiyu Geng ${ }^{2^{*}}$

\begin{abstract}
Alzheimer's disease (AD) is a progressive neurodegenerative disorder and the most common cause for dementia. There are many hypotheses about $A D$, including abnormal deposit of amyloid $\beta(A \beta)$ protein in the extracellular spaces of neurons, formation of twisted fibers of tau proteins inside neurons, cholinergic neuron damage, inflammation, oxidative stress, etc., and many anti-AD drugs based on these hypotheses have been developed. In this review, we will discuss the existing and emerging hypothesis and related therapies.
\end{abstract}

Keywords: Alzheimer's disease - complicated disease - anti-AD drugs, Hypothesis about AD

\section{Background}

Alzheimer's disease (AD) is a progressive neurodegenerative disorder, which is the most common cause for dementia and imposes immense suffering on patients and their families. According to the World Alzheimer Report 2016, there are currently about 46.8 million people suffering with $\mathrm{AD}$ worldwide. The ageing of world population will further compound this problem and lead to a steep increase in the number of $A D$ patients. The numbers of $\mathrm{AD}$ patients are expected to double nearly every 20 years, and thereby the population of AD will reach 74.7 million in 2030 and 131.5 million in 2050 [1]. AD has become the third major cause of disability and death for the elderly, only after cardiovascular and cerebrovascular diseases and malignant tumors.

However, only five drugs have been approved by the FDA to treat AD over the past hundred years since the first AD patient was diagnosed. Not only that, these approved drugs including cholinesterase inhibitors, Nmethyl-D-aspartate (NMDA) receptor antagonist or their combination usually provide temporary and incomplete symptomatic relief accompanied with severe side effects. The marginal benefits were unable to slow the progression of $\mathrm{AD}$. Thus, developing drugs for more effective $\mathrm{AD}$ treatment is in urgent need.

\footnotetext{
* Correspondence: mygeng@simm.ac.cn

${ }^{2}$ State Key Laboratory of Drug Research, Shanghai Institute of Materia Medica, Chinese Academy of Sciences, 555 Zu Chong Zhi Road, Shanghai 201203, People's Republic of China

Full list of author information is available at the end of the article
}

\section{Current hypothesis about $A D$ and anti-AD drug development}

$\mathrm{AD}$ is a complicated disease involving many factors. Due to the complexity of human brains, the lack of reasonable animal models and research tools, the detailed pathogenesis of AD is still unclear so far. Many hypotheses about $\mathrm{AD}$ have been developed, including amyloid $\beta$ (A $\beta)$, Tau, cholinergic neuron damage and oxidative stress, inflammation, etc. Thus, many efforts have been done to develop anti-AD drugs based on these hypotheses.

\section{$A \beta$ cascade hypothesis}

Extracellular deposits of $A \beta$ peptides as senile plaques, intraneuronal neurofibrillary tangles (NFTs), and largescale neuronal loss were the main pathological features of $\mathrm{AD}$. Thus, $\mathrm{A} \beta$ peptides have long been viewed as a potential target for $\mathrm{AD}$ which dominated new drug research during the past twenty years [2]. The most direct strategy in anti-A $\beta$ therapy is to reduce $A \beta$ production by targeting $\beta$ - and $\gamma$-secretase [3]. Safety issues are the overriding problem. For targeting $\gamma$-secretase, undesirable side effects are inevitable due to its physiological substrates, eg. the Notch signaling protein [4-7], which is essential in normal biological process. Similarily, targeting $\beta$-secretase is also challenged for the side effects such as blindness and the large catalytic pocket [8]. More importantly, in sporadic AD cases, the majority of $\mathrm{AD}$ patients do not necessarily have over-producted amyloid precursor protein. Besides, $A \beta$ isoforms could also serve as endogenous positive regulators 
for neurotransmitter release at hippocampal synapses [9]. Thus, inhibiting $A \beta$ production may encounter many challenges.

$\mathrm{A} \beta$ clearance by immunotherapy is the alternative choice. For active $A \beta$-immunotherapy, although the first active AD vaccine (AN1792) developed by ELAN showed some beneficial effects such as less cognitive decline, it was suspended owing to serious side effect, or meningoencephalitis [10-12]. Also, the passive immunotherapy did not do much better than active immunotherapy. Several antibodies targeting $A \beta$ have failed in clinical trials, including bapineuzumab (Pfizer/Johnson \& Johnson) [13, 14], Crenezumab (Genentech) [15, 16], solanezumab (Eli Lilly) [16-18] and ponezumab (Johnson \& Johnson /Pfizer) [19-21]. In addition, although passive immunotherapy could overcome some problems of active immunotherapy, there were still inevitable side effects such as amyloid-related imaging abnormalities [22]. Likewise, the small molecule $A \beta$ binder scyllo-inositol [23] and tramiprosate [24-26] also failed in clinical trials. These failures even cast more doubts on the $A \beta$ theory [27]. Actually, the strategy of targeting only a single functional subregion of $A \beta$ may partly account for these failures [27, 28]. Furthermore, immunotherapy may influence the human immune system, which might cause beneficial or detrimental consequence (such as side effects). However, every cloud has a silver lining. A phase Ib trial of aducanumab (Biogen) showed a positive correlation between brain $A \beta$ levels and disease exacerbation as measured by Clinical Dementia Rating [29-31]. Even the failed phase III EXPEDITION3 trial of solanezumab (Eli Lilly) still demonstrated better performance in Clinical Dementia Rating Sum of Boxes and beneficial impacts on MiniMental State Examination and Activities of Daily Living $[17,18,32,33]$. Thus, despite all kinds of problems, immunotherapy may still be the better approach to modify the extent of neurodegeneration in $\mathrm{AD}$ currently [34].

In fact, the original amyloid cascade hypothesis was that " $A \beta$ is the causative agent in Alzheimer's Disease pathology, and that neurofibrillary tangles, cell loss, vascular damage, and dementia follow as a direct result of this deposition" [35]. After decades of research, although the bulk of data still supports a role for $A \beta$ as the primary initiator of the complex pathogenic cascade in $A D$, more and more evidences indicate that $A \beta$ acts as a trigger in the early disease process and appears to be necessary but not sufficient in the late stage of $\mathrm{AD}$ [36]. Especially, recent rapid progresses in understanding on toxic amyloid assembly and $A \beta$ metabolism associated systemic abnormalities will provide fresh impetus and new opportunities for this interesting approach [37].

\section{Tau hypothesis}

Neurofibrillary tangles, another intracellular hallmark of $\mathrm{AD}$, are composed of tau. Tau is a microtubuleassociated protein working as scaffolding proteins that are enriched in axons. In pathological conditions, tau aggregation will impair axons of neurons and thus cause neurodegeneration. After numerous failures of $A \beta$ targeting drugs for $\mathrm{AD}$, more interests are turning to explore the therapeutic potential of targeting tau, particularly as studies of biomarkers suggest that tau pathology is more closely linked to the progression of AD [38].

Tau undergoes many modifications, including phosphorylation, arginine monomethylation, lysine acetylation, lysine monomethylation, lysine dimethylation, lysine ubiquitylation and serine.

O-linked N-acetylglucosamine (O-GlcNAc) modification [39]. Under pathological conditions, increasing of tau hyperphosphorylation will render the protein aggregation-proned, reduce its affinity for microtubules, and thereby influence neuronal plasticity. Consequently, strategies to target tau involve blocking of tau aggregation, utilizing tau vaccinations, stabilizing microtubules, manipulating kinases and phosphatases that govern tau modifications. However, most of these efforts have failed in clinical trials. For Tau aggregation blockers, TRx0237 failed to show treatment benefits in phase III trials [40]. As for vaccinations, tau-targeted active vaccines (ACI35 and AADvac-1) and passive vaccines (RG6100 and ABBv-8E12) are currently in phase I and II clinical trials [41, 42]. Intravenous immunoglobulin (IVIG), the only passive vaccine in phase III clinical trials, failed to meet the primary end points in patients with mild-tomoderate $\mathrm{AD}$ [42]. Other tau-targeting strategies for $\mathrm{AD}$, including stabilizing microtubules and manipulating kinases and phosphatases, have just been tested in preclinical studies.

In general, tau-targeting therapies remain challenging because of incomplete understanding of $\mathrm{AD}$, lack of robust and sensitive biomarkers for diagnosis and response-monitoring, and the obstruction of blood-brain barrier.

\section{Inflammation hypothesis}

Reactive gliosis and neuroinflammation are hallmarks of AD. Microglia-related pathways were considered to be central to AD risk and pathogenesis, as supported by emerging genetic and transcriptomic studies [43-47]. Increasing evidence demonstrate that microglia emerges as central players in AD. In very early stage, microglia, TREM2 and complement system are responsible for synaptic pruning [48, 49]. The processes of activitydependent and long-term synaptic plasticity are the common and fundamental cellular underpinning of learning 
and memory which may manifest as influence on long term potential [50]. Following that, reactive microglia and astrocytes will surround amyloid plaques and secrete numerous pro-inflammatory cytokines. These events are regarded as an early, prime mover in AD evolution. However, non-steroid anti-inflammatory drugs (NSAIDs) did not show enough benefits in clinic. This is because that the relationship between innate immunity and $\mathrm{AD}$ pathogenesis is complex, and the immune response can be either deleterious or beneficial depending on the context $[47,51,52]$. However, the new observations that PD-1 immune checkpoint blockade reduces the pathology of $\mathrm{AD}$ and improves memory in mouse models of AD [53-55] give us a direction of future researches.

The recent advances in our understanding of the mechanism underlying microglia dysfunction in pruning, regulating plasticity, and neurogenesis are opening up possibilities for new opportunities of $\mathrm{AD}$ therapeutic interventions and diagnosis [56, 57]. Targeting these aberrant microglial functions and thereby returning homeostasis may yield novel paradigms for AD therapies. However, given the complexity and diverse functions of microglia in health and disease, there is a crucial need for new biomarkers reflecting the function of specific microglias $[52,58]$.

\section{Cholinergic and oxidative stress hypothesis}

Acetylcholine (ACh) is an important neurotransmitter used by cholinergic neurons, which has been involved in critical physiological processes, such as attention, learning, memory, stress response, wakefulness and sleep, and sensory information [59-63]. Cholinergic neurons damage was considered to be a critical pathological change that correlated with cognitive impairment in AD. Thus, cholinergic hypothesis was firstly tested with cholinesterase inhibitors in AD treatment. Tacrine, a cholinesterase inhibitor, was the first anti-AD drug available in clinic [64-66] although it was withdrawn from the market in 2012 due to severe side effects. Although inhibiting cholinesterase is a symptomatic relief treatment with marginal benefits, it is currently the most available clinical treatment which gives desperate $\mathrm{AD}$ patients a glimmer of hope. For other neurotransmitter dysfunction, such as Dopamine and 5-hydroxytryptamine, there are some studies about them, but not much as acetylcholine in AD.

Oxidative stress is considered to play an important role in the pathogenesis of AD. Especially, the brain utilizes more oxygen than other tissues and undergoes mitochondrial respiration, which increases the potential for ROS exposure. In fact, AD is highly associated with cellular oxidative stress, including augmentation of protein oxidation, protein nitration, glycoloxidation and lipid peroxidation as well as accumulation of $A \beta$, for $A \beta$ can also induce oxidative stress [67-73]. Thus, the treatment with anti-oxidant compounds would provide protection against oxidative stress and $A \beta$ toxicity in theory. However, oxidative stress is only a single feature of $\mathrm{AD}$, so antioxidant strategy was challenged for its potency to stop the progression of $\mathrm{AD}$ and thus it is proposed as a portion of combination therapy [74, 75].

\section{Glucose hypometabolism}

Glucose hypometabolism is the early pathogenic event in the prodromal phase of $\mathrm{AD}$, and associated with cognitive and functional decline. Early therapeutic intervention before the irreversible degeneration has become a consensus in $\mathrm{AD}$ treatment. Thus, alleviation of glucose hypometabolism was emerged as an attractive strategy of AD treatment. However, most of these therapeutic strategies are targeting mitochondria and bioenergetics, which have shown promise at the preclinical stage but without success in clinical trials [76, 77]. Although no strategies are available to alleviate glucose hypometabolism in clinical, glucose metabolism brain imaging such as ${ }^{18}$ FDG-PET (Positron emision tomography with 2deoxy-2-fluorine-18-fluoro-D-glucose) has become a valuable indicator for diagnosis of neurodegenerative diseases that cause dementia, including AD [78].

Up to now, there're no effective treatments for changing the course of AD. Confronting these difficulties, we should get deeper understandings about these hypotheses, and meanwhile we should renovate our knowledge about $\mathrm{AD}$ and develop new hypothesis.

\section{New pathway to AD}

$\mathrm{AD}$ is conventionally regarded as a central nervous system (CNS) disorder. However, increasing experimental, epidemiological and clinical evidences have suggested that manifestations of $\mathrm{AD}$ extend beyond the brain. Most notably, research over the past few years reveals that the gut microbiome (GMB) has a profound impact on the formation of the blood-brain barrier, myelination, neurogenesis, and microglia maturation [79-84]. In particular, results from germ-free animals and animals exposed to pathogenic microbial infections, antibiotics, probiotics, or fecal microbiota transplantation showed that gut microbiota modulates many aspects of animal behaviors, suggesting a role for the gut microbiota in host cognition or AD-related pathogenesis [85-88]. The underlying mechanisms of gut microbiota influencing brain involve the communication through immune system, the endocrine system, the vague nerve, and the bacteria-derived metabolites.

\section{Immune pathway}

The intestinal mucosal lymphoid tissue contains $70 \%$ $80 \%$ of the immune cells in the whole body, and is 
considered to be the largest and most important human immune organs. It is also the first line of host defense against pathogens. The human gut contains a large, diverse and dynamic enteric microbiota, including more than 100 trillion microorganisms from at least 1000 distinct species. There's a complex relationship between intestinal mucosal immune system and intestinal microbiota. Thus, gut microbiota induced immunomodulation is emerging as an important pathway that influences $\mathrm{AD}$ [89].

Gut microbiota can influence brain through immune system in several ways. Firstly, intestinal microbiome can induce cytokines secretion, which enter the circulatory system, pass through blood brain barrier, and directly affect the brain function. For instance, perivascular macrophages and cerebral small vessel epithelial cells can receive the intestinal microbiome produced IL-1 signal and affect central nervous system. Also, gut microbes can activate Toll-like receptors of the brain immune cells (such as microglia) through microbes associated molecular patterns (MAMP). MAMPs can either directly bind to intestinal epithelial cells or infiltrate to the intestine lamina propria to activate lymphocytes, promoting the release of pro-inflammatory cytokines, which further cause subsequent inflammation in brain. Secondly, gut microbes can produce metabolites such as short-chainfatty acids (SCFAs), gamma-aminobutyric acid (GABA) and 5-HT precursors, which could also travel to the brain via circulatory systems or signal through intestinal epithelials to produce cytokines or neurotransmitters that activate vagus nerve. Thirdly, gut microbes can activate enteroendocrine cells to produce 5 -HT, which affect the brain through neuroimmune pathways.

In addition to changing the functions of the immune system, such as through secretion of inflammatory factors or anti-inflammatory factors, intestinal microbiome can also affect the development and composition of immune system. For example, in germ-free mice, isolated lymphoid follicles in gut associated lymphoid tissue are unable to mature, and lymphocytes that are able to secrete IgA in the intestinal epithelium decreased [89-92]. For immune system in brain, the deletion of gut microbiota in germ-free mice have global influence on the cell proportions and maturation of microglia in the brain, and thus affect the properties and phenotype of microglia, as compared to conventionally colonized controls [93]. Similar results were obtained in antibiotic treated mice. Other research also demonstrates that the number of $\mathrm{T}$ regulatory cells and $\mathrm{T}$ helper lymphocytes ( $\mathrm{T}$ helper 17, Th17) are significantly reduced in the germ free mouse, indicating the regulatory effects of intestinal microbiome on $\mathrm{T}$ cell composition, while microbiome tansplant to germ free mice can modify these variations and restore normal immune function [94, 95]. All these modulations of gut microbiota may have direct and indirect effects on AD development and progression.

\section{Endocrine pathway and the vagus nerve}

The gut is also the largest endocrine organ in the body. Gut microbiota can regulate secretion of many hormones from intestinal endocrine cells, such as corticosterone and adrenal hormones, and thus establish the information exchange between the intestines and the brain. For example, the intestinal microbiome can affect the secretion of serotonin and regulate brain emotional activities [96, 97]; intestinal microbial metabolism can also produce a variety of neurotransmitters, such as dopamine, GABA, acetylcholine and melatonin, which are transmitted to central nervous system through the vagus nerve [98]. Besides transporting these signal substances, the vagus nerve itself plays an important role in inflammation and depression [99]. The vagus nerve can influence the gastrointestinal tract, orchestrate the complex interactions between central and peripheral neural control mechanisms [100]. The stimulation of vagus nerve is able to regulate mood, and the immune system, suggesting the therapeutic potential of vagus nerve modulation to attenuate the pathophysiological changes and restore homeostasis [98-103].

\section{Bacteria-derived metabolites}

Generation of essential nutrients for host physiology, such as vitamins and other cofactors, is an important physiological function of the gut microbiota [104]. The metabolites of microbiome, such as SCFAs including acetate, butyrate, and propionate, are able to modulate peripheral and central pathologic processes [105]. For example, butyrate is effective in reducing inflammation and pain. Once in the brain, acetate is able to alter the level of the neurotransmitters glutamate, glutamine, and GABA, as well as increases anorectic neuropeptide expression [106]. In addition, the gut microbiota can secrete large amounts of amyloids and lipopolysaccharides, which might contribute to the modulation of signaling pathways, the production of proinflammatory cytokines associated with $\mathrm{AD}$ pathogenesis and $\mathrm{A} \beta$ deposition [107-109].

In fact, microbiota-gut-brain axis has been established and a disturbed gut microbiota has been incriminated in many neurodegenerative diseases in animal and translational models. In theory, a role for the microbiota-gutbrain axis is highly plausible. However, the theoretical basis for the use of microbiota-directed therapies in neurodegenerative disorders still needs supports from high-quality clinical trials [110]. To date, only a few studies directly focused on the gut microbiota and $\mathrm{AD}$ [111, 112], and studies on AD patients is particullarly deficient. A recent research from human showed an increase in the abundance of a pro-inflammatory GMB 
taxon and a reduction in the abundance of an antiinflammatory taxon are possibly associated with a peripheral inflammatory state in patients with cognitive impairment and brain amyloidosis. It is important for the research of gut microbiota and AD. However, further investigations are still necessary to explore the possible causal relation between GMB-related inflammation and amyloidosis [111]. The comprehensive understanding of these underlying mechanisms may provide new insights into these novel therapeutic strategies for $\mathrm{AD}$. In particular, based on the gut microbiota hypothesis, Chinese traditional medicine and probiotic bacteria may play a more important role in therapy [113].

\section{Conclusions}

Nowadays, new technologies are making it possible to get to know enough pathologic details of disease. More importantly, scientists are beginning to treat $\mathrm{AD}$ as a systemic disease and they are paying more attention to the correlation between brain and other organs [47, 89, 114]. Perhaps, for complicated disease such as AD, researches and therapies should be based on the principle that combined reductionism with holism, and great efforts should be made to search the fundamental laws of AD by means of multi-scale modeling and efficient numeric assessment. Maybe, just like Chinese traditional medicine [115], combination treatments or systematic therapy will be a final way out.

\section{Abbreviations}

AD: Alzheimer's disease; AB: Amyloid $\beta$; CNS: Central nervous system; CSF1R: Colony stimulating factor 1 receptor; GABA: Gamma-aminobutyric acid; IVIG: Intravenous immunoglobulin; MAMP: Microbes associated molecular patterns; NFTs: Neurofibrillary tangles; NMDA: N-methyl-Daspartate; NSAIDs: Non-steroid anti-inflammatory drugs; O-GIcNAc: O-linked $\mathrm{N}$-acetylglucosamine; SCFAs: Short-chain-fatty acids; Th17: T helper lymphocytes 17

\section{Acknowledgements}

Not applicable.

\section{Funding}

This review was supported by "Personalized Medicines-Molecular Signature based Drug Discovery and Development", Strategic Priority Research program of Sciences, Grants No. XDA12040101.

\section{Availability of data and materials}

Not applicable.

\section{Authors' contributions}

All authors read and approved the final manuscript. XD: Forming the concept, drafting and revising the manuscript; XW: Revising the immune pathway; MG: Revising and approving the manuscript.

\section{Ethics approval and consent to participate}

Not relevant.

\section{Consent for publication}

Not applicable.

\section{Competing interests}

The authors declare they have no competing interests.

\section{Author details}

'Shanghai GreenValley Pharmaceutical Co., Ltd., 421 Newton Road, Shanghai 201203, People's Republic of China. ${ }^{2}$ State Key Laboratory of Drug Research, Shanghai Institute of Materia Medica, Chinese Academy of Sciences, $555 \mathrm{Zu}$ Chong Zhi Road, Shanghai 201203, People's Republic of China.

Received: 7 November 2017 Accepted: 18 January 2018

Published online: 30 January 2018

\section{References}

1. Ali G-C, Guerchet M, Wu Y-T, Prince M, Prina M. Chapter 2: The global prevalence of dementia. In: Prince M, Guerchet M, Ali G-C, Wu Y-T, Prina M, editors. The Global Impact of Dementia. An analysis of prevalence, incidence, cost and trends. London: Alzheimer's Disease International (ADI); 2015. p. 10-29.

2. Cummings J, et al. Drug development in Alzheimer's disease: the path to 2025. Alzheimers Res Ther. 2016;8:39.

3. Vassar R, Citron M. Abeta-generating enzymes: recent advances in beta- and gamma-secretase research. Neuron. 2000;27(3):419-22.

4. van Es JH, et al. Notch/gamma-secretase inhibition turns proliferative cells in intestinal crypts and adenomas into goblet cells. Nature. 2005;435(7044): 959-63.

5. Olry A, et al. Generation and characterization of mutant cell lines defective in gamma-secretase processing of notch and amyloid precursor protein. J Biol Chem. 2005;280(31):28564-71.

6. Tarassishin $L$, et al. Processing of notch and amyloid precursor protein by gamma-secretase is spatially distinct. Proc Natl Acad Sci U S A. 2004;101(49): 17050-5.

7. Sastre $M$, et al. Presenilin-dependent gamma-secretase processing of betaamyloid precursor protein at a site corresponding to the S3 cleavage of notch. EMBO Rep. 2001;2(9):835-41.

8. Klaver DW, et al. Is BACE1 a suitable therapeutic target for the treatment of Alzheimer's disease? Current strategies and future directions. Biol Chem. 2010;391(8):849-59.

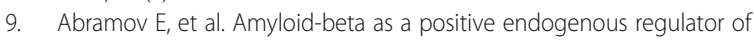
release probability at hippocampal synapses. Nat Neurosci. 2009;12(12): 1567-76.

10. Gilman S, et al. Clinical effects of Abeta immunization (AN1792) in patients with AD in AN interrupted trial. Neurology. 2005;64(9):1553-62.

11. Bayer AJ, et al. Evaluation of the safety and immunogenicity of synthetic Abeta42 (AN1792) in patients with AD. Neurology. 2005;64(1):94-101.

12. Holmes $C$, et al. Long-term effects of Abeta42 immunisation in Alzheimer's disease: follow-up of a randomised, placebo-controlled phase I trial. Lancet. 2008;372(9634):216-23.

13. Laskowitz DT, Kolls BJ. A phase 2 multiple ascending dose trial of bapineuzumab in mild to moderate Alzheimer disease. Neurology. 2010; 74(24):2026. author reply 2026-7

14. Salloway $\mathrm{S}$, et al. A phase 2 multiple ascending dose trial of bapineuzumab in mild to moderate Alzheimer disease. Neurology. 2009;73(24):2061-70.

15. Ultsch M, et al. Structure of Crenezumab complex with Abeta shows loss of beta-hairpin. Sci Rep. 2016;6:39374.

16. Bouter $Y$, et al. Abeta targets of the biosimilar antibodies of Bapineuzumab, Crenezumab, Solanezumab in comparison to an antibody against Ntruncated Abeta in sporadic Alzheimer disease cases and mouse models. Acta Neuropathol. 2015;130(5):713-29.

17. The Lancet N. Solanezumab: too late in mild Alzheimer'ss disease? Lancet Neurol. 2017;16(2):97.

18. Gandy S, Sano M. Alzheimer disease: Solanezumab-prospects for meaningful interventions in AD? Nat Rev Neurol. 2015;11(12):669-70.

19. Landen JW, et al. Safety and pharmacology of a single intravenous dose of ponezumab in subjects with mild-to-moderate Alzheimer disease: a phase I, randomized, placebo-controlled, double-blind, dose-escalation study. Clin Neuropharmacol. 2013;36(1):14-23.

20. Burstein AH, et al. Safety and pharmacology of ponezumab (PF-04360365) after a single 10-minute intravenous infusion in subjects with mild to moderate Alzheimer disease. Clin Neuropharmacol. 2013;36(1):8-13.

21. La Porte SL, et al. Structural basis of C-terminal beta-amyloid peptide binding by the antibody ponezumab for the treatment of Alzheimer's disease. J Mol Biol. 2012;421(4-5):525-36.

22. Carlson $C$, et al. Amyloid-related imaging abnormalities from trials of solanezumab for Alzheimer's disease. Alzheimers Dement (Amst). 2016; 2:75-85. 
23. Salloway S, et al. A phase 2 randomized trial of ELND005, scyllo-inositol, in mild to moderate Alzheimer disease. Neurology. 2011;77(13):1253-62.

24. Aisen PS, et al. Tramiprosate in mild-to-moderate Alzheimer's disease - a randomized, double-blind, placebo-controlled, multi-centre study (the Alphase study). Arch Med Sci. 2011;7(1):102-11.

25. Greenberg SM, et al. A phase 2 study of tramiprosate for cerebral amyloid angiopathy. Alzheimer Dis Assoc Disord. 2006;20(4):269-74.

26. Gervais F, et al. Targeting soluble Abeta peptide with Tramiprosate for the treatment of brain amyloidosis. Neurobiol Aging. 2007;28(4):537-47.

27. Karran E, Mercken M, De Strooper B. The amyloid cascade hypothesis for Alzheimer's disease: an appraisal for the development of therapeutics. Nat Rev Drug Discov. 2011;10(9):698-712.

28. Nie Q, Du XG, Geng MY. Small molecule inhibitors of amyloid beta peptide aggregation as a potential therapeutic strategy for Alzheimer's disease. Acta Pharmacol Sin. 2011:32(5):545-51.

29. Sevigny J, et al. Addendum: the antibody aducanumab reduces Abeta plaques in Alzheimer's disease. Nature. 2017:546(7659):564.

30. Sevigny J, et al. The antibody aducanumab reduces Abeta plaques in Alzheimer's disease. Nature. 2016;537(7618):50-6.

31. Patel KR. Biogen's aducanumab raises hope that Alzheimer's can be treated at its source. Manag Care. 2015;24(6):19.

32. Karran E. Recent trial shows that solanezumab has disease modifying effects. BMJ. 2015:351:h4528.

33. Siemers ER, et al. Phase 3 solanezumab trials: secondary outcomes in mild Alzheimer's disease patients. Alzheimers Dement. 2016;12(2):110-20.

34. Barrera-Ocampo A, Lopera F. Amyloid-beta immunotherapy: the hope for Alzheimer disease? Colomb Med (Cali). 2016;47(4):203-12.

35. Hardy JA, Higgins GA. Alzheimer's disease: the amyloid cascade hypothesis. Science. 1992;256(5054):184-5.

36. Musiek ES, Holtzman DM. Three dimensions of the amyloid hypothesis: time, space and 'wingmen'. Nat Neurosci. 2015;18(6):800-6.

37. Wang J, et al. A systemic view of Alzheimer disease - insights from amyloidbeta metabolism beyond the brain. Nat Rev Neurol. 2017;13(10):612-23.

38. Brier MR, et al. Tau and Abeta imaging, CSF measures, and cognition in Alzheimer's disease. Sci Transl Med. 2016;8(338):338ra66.

39. Morris $M$, et al. Tau post-translational modifications in wild-type and human amyloid precursor protein transgenic mice. Nat Neurosci. 2015;18(8):1183-9.

40. Gauthier S, et al. Efficacy and safety of tau-aggregation inhibitor therapy in patients with mild or moderate Alzheimer's disease: a randomised, controlled, double-blind, parallel-arm, phase 3 trial. Lancet. 2016;388(10062):2873-84

41. Novak $P$, et al. Safety and immunogenicity of the tau vaccine AADvac1 in patients with Alzheimer's disease: a randomised, double-blind, placebocontrolled, phase 1 trial. Lancet Neurol. 2017;16(2):123-34.

42. Li C, Gotz J. Tau-based therapies in neurodegeneration: opportunities and challenges. Nat Rev Drug Discov. 2017;

43. Zhang B, et al. Integrated systems approach identifies genetic nodes and networks in late-onset Alzheimer's disease. Cell. 2013;153(3):707-20.

44. Guerreiro R, et al. TREM2 variants in Alzheimer's disease. N Engl J Med. 2013;368(2):117-27.

45. Song $W$, et al. Alzheimer's disease-associated TREM2 variants exhibit either decreased or increased ligand-dependent activation. Alzheimers Dement. 2017;13(4):381-7.

46. Colonna M, Wang Y. TREM2 variants: new keys to decipher Alzheimer disease pathogenesis. Nat Rev Neurosci. 2016;17(4):201-7.

47. Bolos M, Perea JR, Avila J. Alzheimer's disease as an inflammatory disease. Biomol Concepts. 2017;8(1):37-43.

48. Hong S, Dissing-Olesen L, Stevens B. New insights on the role of microglia in synaptic pruning in health and disease. Curr Opin Neurobiol. 2016;36:128-34.

49. Paolicelli RC, et al. Synaptic pruning by microglia is necessary for normal brain development. Science. 2011:333(6048):1456-8.

50. Bliss TV, Collingridge GL, Morris RG. Synaptic plasticity in health and disease: introduction and overview. Philos Trans R Soc Lond Ser B Biol Sci. 2014;369(1633):20130129

51. Chen J, et al. Inhibition of AGEs/RAGE/rho/ROCK pathway suppresses nonspecific neuroinflammation by regulating BV2 microglial M1/M2 polarization through the NF-kappaB pathway. J Neuroimmunol. 2017;305:108-14.

52. Hirbec H E, Noristani HN, Perrin FE. Microglia Responses in Acute and Chronic Neurological Diseases: What Microglia-Specific Transcriptomic Studies Taught (and did Not Teach) Us. Front Aging Neurosci. 2017:9:227.
53. Baruch K, et al. PD-1 immune checkpoint blockade reduces pathology and improves memory in mouse models of Alzheimer's disease. Nat Med. 2016;22(2):135-7.

54. Saresella M, et al. A potential role for the PD1/PD-L1 pathway in the neuroinflammation of Alzheimer's disease. Neurobiol Aging. 2012;33(3):624 e11-22.

55. Saresella M, et al. PD1 negative and PD1 positive CD4+ T regulatory cells in mild cognitive impairment and Alzheimer's disease. J Alzheimers Dis. 2010;21(3):927-38

56. Jevtic $\mathrm{S}$, et al. The role of the immune system in Alzheimer disease: etiology and treatment. Ageing Res Rev. 2017;40:84-94.

57. McGeer PL, McGeer EG. Targeting microglia for the treatment of Alzheimer's disease. Expert Opin Ther Targets. 2015;19(4):497-506.

58. Salter MW, Stevens B. Microglia emerge as central players in brain disease. Nat Med. 2017:23(9):1018-27.

59. Hasselmo ME, Anderson BP, Bower JM. Cholinergic modulation of cortical associative memory function. J Neurophysiol. 1992;67(5):1230-46.

60. Fine $A$, et al. Learning impairments following injection of a selective cholinergic immunotoxin, ME20.4 lgG-saporin, into the basal nucleus of Meynert in monkeys. Neuroscience. 1997;81(2):331-43.

61. Sarter M, Bruno JP. Cognitive functions of cortical acetylcholine: toward a unifying hypothesis. Brain Res Brain Res Rev. 1997;23(1-2):28-46.

62. Miranda Ml, Bermudez-Rattoni F. Reversible inactivation of the nucleus basalis magnocellularis induces disruption of cortical acetylcholine release and acquisition, but not retrieval, of aversive memories. Proc Natl Acad Sci U S A. 1999:96(11):6478-82.

63. Haam J, Yakel JL. Cholinergic modulation of the hippocampal region and memory function. J Neurochem. 2017;142(Suppl 2):111-21.

64. Brinkman SD, Gershon S. Measurement of cholinergic drug effects on memory in Alzheimer's disease. Neurobiol Aging. 1983;4(2):139-45.

65. Summers WK, et al. Oral tetrahydroaminoacridine in long-term treatment of senile dementia, Alzheimer type. N Engl J Med. 1986;315(20):1241-5.

66. Summers WK, et al. Use of THA in treatment of Alzheimer-like dementia: pilot study in twelve patients. Biol Psychiatry. 1981;16(2):145-53.

67. Cheignon $C$, et al. Oxidative stress and the amyloid beta peptide in Alzheimer's disease. Redox Biol. 2018;14:450-64.

68. Sultana R, Butterfield DA. Redox proteomics studies of in vivo amyloid betapeptide animal models of Alzheimer's disease: insight into the role of oxidative stress. Proteomics Clin Appl. 2008;2(5):685-96.

69. Butterfield DA, et al. Roles of amyloid beta-peptide-associated oxidative stress and brain protein modifications in the pathogenesis of Alzheimer's disease and mild cognitive impairment. Free Radic Biol Med. 2007:43(5):658-77.

70. Mohmmad Abdul $\mathrm{H}$, et al. Mutations in amyloid precursor protein and presenilin-1 genes increase the basal oxidative stress in murine neuronal cells and lead to increased sensitivity to oxidative stress mediated by amyloid beta-peptide (1-42), $\mathrm{HO}$ and kainic acid: implications for Alzheimer's disease. J Neurochem. 2006;96(5):1322-35.

71. Gibson GL, Allsop D, Austen BM. Induction of cellular oxidative stress by the beta-amyloid peptide involved in Alzheimer's disease. Protein Pept Lett. 2004;11(3):257-70.

72. Butterfield DA, et al. Amyloid beta-peptide and amyloid pathology are central to the oxidative stress and inflammatory cascades under which Alzheimer's disease brain exists. J Alzheimers Dis. 2002;4(3):193-201.

73. Butterfield DA, Lauderback CM. Lipid peroxidation and protein oxidation in Alzheimer's disease brain: potential causes and consequences involving amyloid beta-peptide-associated free radical oxidative stress. Free Radic Biol Med. 2002;32(11):1050-60

74. Persson T, Popescu BO, Cedazo-Minguez A. Oxidative stress in Alzheimer's disease: why did antioxidant therapy fail? Oxidative Med Cell Longev. 2014;2014:427318.

75. Teixeira J, et al. Alzheimer's disease and antioxidant therapy: how long how far? Curr Med Chem. 2013;20(24):2939-52

76. Caldwell CC, Yao J, Brinton RD. Targeting the prodromal stage of Alzheimer's disease: bioenergetic and mitochondrial opportunities. Neurotherapeutics. 2015;12(1):66-80.

77. Daulatzai MA. Cerebral hypoperfusion and glucose hypometabolism: key pathophysiological modulators promote neurodegeneration, cognitive impairment, and Alzheimer's disease. J Neurosci Res. 2017;95(4):943-72.

78. Nasrallah I, Dubroff J. An overview of PET neuroimaging. Semin Nucl Med. 2013;43(6):449-61. 
79. Deverman BE, Patterson PH. Cytokines and CNS development. Neuron. 2009;64(1):61-78.

80. Stephan AH, Barres BA, Stevens B. The complement system: an unexpected role in synaptic pruning during development and disease. Annu Rev Neurosci. 2012;35:369-89.

81. Lui $\mathrm{H}$, et al. Progranulin deficiency promotes circuit-specific synaptic pruning by microglia via complement activation. Cell. 2016;165(4):921-35.

82. Elmer BM, McAllister AK. Major histocompatibility complex class I proteins in brain development and plasticity. Trends Neurosci. 2012;35(11):660-70.

83. Erickson MA, Dohi K, Banks WA. Neuroinflammation: a common pathway in CNS diseases as mediated at the blood-brain barrier. Neuroimmunomodulation. 2012;19(2):121-30.

84. Banks WA. The blood-brain barrier in neuroimmunology: Tales of separation and assimilation. Brain Behav Immun. 2015;44:1-8.

85. Collins SM, Surette M, Bercik P. The interplay between the intestinal microbiota and the brain. Nat Rev Microbiol. 2012;10(11):735-42.

86. Cryan JF, Dinan TG. Mind-altering microorganisms: the impact of the gut microbiota on brain and behaviour. Nat Rev Neurosci. 2012;13(10):701-12.

87. Sampson TR, Mazmanian SK. Control of brain development, function, and behavior by the microbiome. Cell Host Microbe. 2015;17(5):565-76.

88. Bhattacharjee S, Lukiw WJ. Alzheimer's disease and the microbiome. Front Cell Neurosci. 2013;7:153

89. Belkaid Y, Harrison OJ. Homeostatic immunity and the microbiota. Immunity. 2017:46(4):562-76.

90. Belkaid Y, Hand TW. Role of the microbiota in immunity and inflammation. Cell. 2014;157(1):121-41.

91. Tomkovich S, Jobin C. Microbiota and host immune responses: a love-hate relationship. Immunology. 2016;147(1):1-10.

92. Agace WW, McCoy KD. Regionalized development and maintenance of the intestinal adaptive immune landscape. Immunity. 2017;46(4):532-48.

93. Erny D, et al. Host microbiota constantly control maturation and function of microglia in the CNS. Nat Neurosci. 2015;18(7):965-77.

94. Ivanov II, et al. Induction of intestinal Th17 cells by segmented filamentous bacteria. Cell. 2009;139(3):485-98.

95. Sudo N, et al. Postnatal microbial colonization programs the hypothalamicpituitary-adrenal system for stress response in mice. J Physiol. 2004;558(Pt 1):263-75.

96. O'Mahony SM, et al. Serotonin, tryptophan metabolism and the brain-gutmicrobiome axis. Behav Brain Res. 2015;277:32-48.

97. Praveen V, Praveen S. Microbiome-gut-brain Axis: a pathway for improving brainstem serotonin homeostasis and successful autoresuscitation in SIDS-A novel hypothesis. Front Pediatr. 2016:4:136.

98. Jiang $X G$, et al. Research progress in anti-inflammation of vagus nerve and neurotransmitter ach. Zhongguo Wei Zhong Bing Ji Jiu Yi Xue. 2003;15(1):59-61.

99. Das UN. Vagus nerve stimulation, depression, and inflammation. Neuropsychopharmacology. 2007;32(9):2053-4.

100. Browning KN, Verheijden S, Boeckxstaens GE. The Vagus nerve in appetite regulation, mood, and intestinal inflammation. Gastroenterology. 2017;152(4):730-44.

101. de Haan JJ, et al. Exploring the link between inflammation and the vagus nerve. J Intern Med. 2010;267(1):130-1.

102. Hoeger $S$, et al. Modulation of brain dead induced inflammation by vagus nerve stimulation. Am J Transplant. 2010;10(3):477-89.

103. Mirakaj $V$, et al. Vagus nerve controls resolution and pro-resolving mediators of inflammation. J Exp Med. 2014;211(6):1037-48.

104. Gordon Jl, et al. The human gut microbiota and undernutrition. Sci Transl Med. 2012;4(137):137ps12.

105. Mitchell RW, et al. Fatty acid transport protein expression in human brain and potential role in fatty acid transport across human brain microvessel endothelial cells. J Neurochem. 2011;117(4):735-46.

106. Frost $\mathrm{G}$, et al. The short-chain fatty acid acetate reduces appetite via a central homeostatic mechanism. Nat Commun. 2014:5:3611.

107. Zhao Y, Dua P, Lukiw WJ. Microbial sources of amyloid and relevance to Amyloidogenesis and Alzheimer's disease (AD). J Alzheimers Dis Parkinsonism. 2015;5(1):177

108. Schwartz K, Boles BR. Microbial amyloids-functions and interactions within the host. Curr Opin Microbiol. 2013;16(1):93-9.

109. Hill JM, Lukiw WJ. Microbial-generated amyloids and Alzheimer's disease (AD). Front Aging Neurosci. 2015;7:9.
110. Quigley EMM. Microbiota-brain-gut Axis and neurodegenerative diseases. Curr Neurol Neurosci Rep. 2017;17(12):94.

111. Cattaneo A, et al. Association of brain amyloidosis with pro-inflammatory gut bacterial taxa and peripheral inflammation markers in cognitively impaired elderly. Neurobiol Aging. 2017:49:60-8.

112. Park AM, et al. Helicobacter pylori and gut microbiota in multiple sclerosis versus Alzheimer's disease: 10 pitfalls of microbiome studies. Clin Exp Neuroimmunol. 2017:8(3):215-32.

113. Chen $\mathrm{F}$, et al. Could the gut microbiota reconcile the oral bioavailability conundrum of traditional herbs? J Ethnopharmacol. 2016;179:253-64.

114. Zheng $X$, et al. Thinking outside the brain for cognitive improvement: Is peripheral immunomodulation on the way? Neuropharmacology. 2015;96(Pt A):94-104.

115. Yang WT, et al. Chinese herbal medicine for Alzheimer's disease: clinical evidence and possible mechanism of neurogenesis. Biochem Pharmacol. 2017;141:143-55.

\section{Submit your next manuscript to BioMed Central and we will help you at every step:}

- We accept pre-submission inquiries

- Our selector tool helps you to find the most relevant journal

- We provide round the clock customer support

- Convenient online submission

- Thorough peer review

- Inclusion in PubMed and all major indexing services

- Maximum visibility for your research

Submit your manuscript at www.biomedcentral.com/submit 